\title{
Noninvasive Monitoring and Neurointerventional Management of Idiopathic Intracranial Hypertension
}

\author{
Matthew Lang ${ }^{1}$ Sunu Mathew ${ }^{1} \quad$ Alon Harris $^{1, \odot} \quad$ Juan Tejada ${ }^{2} \quad$ Jerry M. Kovoor ${ }^{2, \odot}$ \\ ${ }^{1}$ Department of Ophthalmology, Indiana University School of \\ Medicine, Indianapolis, Indiana, United States \\ 2Department of Radiology, Indiana University School of Medicine, \\ Indianapolis, Indiana, United States

\begin{abstract}
Address for correspondence Jerry Mathew Elias Kovoor, MBBS, DNB, Department of Radiology, Indiana University School of Medicine, 720 Eskenazi Ave., Indianapolis, IN 46202, United States (e-mail: jkovoor@iu.edu).
\end{abstract}

J Clin Interv Radiol ISVIR 2020;4:42-50

\begin{abstract}
Idiopathic intracranial hypertension $(\mathrm{IIH})$ is characterized by isolated rise in intracranial pressure (ICP) leading to chronic, debilitating headaches, tinnitus, and vision loss. Con-

Keywords

- idiopathic intracranial hypertension

- optic nerve sheath

- transcranial Doppler ultrasonography

- optical coherence tomography

- electroretinography

- ventriculoperitoneal shunt

- lumboperitoneal shunt

- venous sinus stenting ventional diagnostics and monitoring primarily require the use of invasive procedure like lumbar puncture to measure ICP, while traditional management strategies involve weight reduction and medical treatment with acetazolamide. In an effort to reduce the need for invasive procedures, noninvasive methods of ICP monitoring such as optic nerve sheath diameter measurements and two-depth transcranial Doppler ultrasonography have been developed. In cases of refractory and fulminant IIH, surgical management strategies such as optic nerve sheath fenestration (ONSF), ventriculoperitoneal (VP) and lumboperitoneal (LP) shunting, and transverse venous sinus stenting are used to relieve symptoms via ICP reduction. While ONSF and VP/LP shunting exhibit utility primarily for the treatment of vision loss and headache, respectively, venous sinus stenting may prove to be an effective option in the management of all symptoms of $\mathrm{IIH}$. Most importantly, consideration of the patient's individual symptoms and values should be taken into account when choosing the optimal surgical management strategy for patients with IIH.
\end{abstract}

\section{Introduction}

The term idiopathic intracranial hypertension (IIH) refers to a condition of elevated intracranial pressure (ICP) without a detectable cause. IIH is a relatively rare condition with an incidence of 1.2 per 100,000 people per year. More specifically, young, obese females are disproportionately affected by $\mathrm{IIH}$ with incidence rates as high as 10.3 per 100,000 people annually among the female population aged between 14 and $45 .{ }^{1}$ Common presenting symptoms of IIH include headache, vomiting, tinnitus, transient visual abnormalities, papilledema, and vision loss. ${ }^{2}$ Vision loss in IIH, specifically visual field defect, is often long term and can be permanent, making it the primary focus of monitoring and treatment strategies. Visual acuity can also be affected in patients with IIH, especially in cases of fulminant IIH associated with severe papilledema and optic neuropathy. ${ }^{3}$ Visual function in medically treated patients with IIH has been demonstrated to improve overtime ${ }^{4-6}$; however, permanent damage is possible in cases of fulminant $\mathrm{IIH}^{7}$

While there is currently no consensus regarding an established pathophysiologic cause of $\mathrm{IIH}$, a variety of theories have been proposed. One such theory suggests that aldosterone excess, as seen in obese patients, acts at the mineralocorticoid receptor of the choroid plexus resulting in increased cerebral spinal fluid (CSF) production. Another theory proposes that estrogen or retinoic acid may be affecting outflow resistance of the CSF. ${ }^{8}$ Transverse venous sinus stenosis, which has been identified in over $90 \%$ of $\mathrm{IIH}$ patients, ${ }^{9}$ is also a proposed contributor to elevated ICP in IIH patients and is of particular relevance due to the growing use of venous sinus stent procedures for ICP reduction in patients with IIH.
License terms

(우 (1) $\ominus \circledast$ 
Due to the unclear pathophysiology of IIH and the frequent occurrence of new findings in the literature, the diagnostic criteria for IIH have been revised multiple times since Walter Dandy first developed a set of criteria in 1937. Most recently, the criteria have been modified by the American Academy of Neurology as well as for the Idiopathic Intracranial Hypertension Treatment Trial (IIHTT). Conventional diagnosis requires physical symptoms associated with raised ICP, absence of localized neurological or radiographic findings, and opening pressure greater than $250 \mathrm{~mm} \mathrm{H}_{2} \mathrm{O}$ (normal reference range: 60-250 $\mathrm{mm} \mathrm{H}_{2} \mathrm{O}^{10}$ ) with normal CSF composition detected by lumbar puncture. More recently, radiographic findings such as transverse venous stenosis and empty sella have been incorporated into the diagnosis of IIH; yet, these findings are not sufficient on their own to make a definitive diagnosis. ${ }^{11,12}$ Additionally, while it does not replace the gold standard method of invasive ICP detection by lumbar puncture, noninvasive measurement of ICP has been made possible by emerging techniques such as measurement of optic nerve sheath diameter (ONSD) as well as two-depth transcranial Doppler (TCD) ultrasonography.

When managing patients with $\mathrm{IIH}$, first-line treatment strategy involves weight reduction, often combined with the use of the carbonic anhydrase inhibitor acetazolamide, to reduce the production of CSF and consequently lower ICP. ${ }^{13,14}$ A reduction of $10 \%$ of current body weight with a target of 1 to $2 \mathrm{lbs}$ per week has been shown to be effective in improvement of symptoms. ${ }^{15}$ The effects of acetazolamide have been proven efficacious in improving visual field, CSF opening pressure, and papilledema by the IIHTT, the first and only randomized, double-blinded control trial prospectively studying the treatment of IIH. While the results of the IIHTT are promising, use of acetazolamide failed to demonstrate improvement in headaches and transient visual obscurations and the improvements due to acetazolamide use were only demonstrated in patients with mild vision loss. ${ }^{16}$ For IIH patients with more severe symptoms or rapidly progressing vision loss, surgical procedures are necessary to prevent permanent loss of vision. Optic nerve sheath fenestration (ONSF), CSF shunting, and transverse venous sinus stenting are the current surgical methods used to reduce ICP and provide symptomatic relief in patients with IIH. ONSF has traditionally been demonstrated to be most effective in improving visual function, while CSF shunting has been more closely associated with headache relief. ${ }^{17,18}$ The emergence of transverse venous sinus stenting, however, may challenge the more conventional surgical treatment approaches.

In an era of high cost healthcare technology and development of a more personalized approach to patient care, it is critical to establish diagnostic and treatment options that can be applied to each individual without the need for repeat procedures or extraneous expenditures due to surgical complications. The current review, therefore, provides an up-to-date and comprehensive summary of the evolving noninvasive diagnostic and monitoring strategies for IIH as well as an investigative comparison of the efficacy, safety, and cost of the major surgical procedures used to manage symptoms in patients with IIH.

\section{Methods}

PubMed searches were conducted through July 2019 with the following keywords: pseudotumor cerebri, optic nerve sheath, TCD ultrasonography, optical coherence tomography, electroretinography, ventriculoperitoneal (VP) shunt, lumboperitoneal (LP) shunt, and venous sinus stenting. References from all relevant articles were also reviewed to capture all pertinent articles. Threshold, sensitivity, and specificity data of noninvasive ICP monitoring techniques was compiled and ranges were used to summarize data from a total of 14 studies using ONSD ( 3 meta-analyses and 5 prospective observational studies, 5 retrospective observational studies) and a total of 5 prospective observational studies using two-depth TCD data describing improvement in vision, papilledema, and headache due to neurointerventional management strategies was piled and ranges were used to summarize data from a total of 7 meta-analyses that each reported pooled data for one, two, or all three neurointerventional management strategies for IIH. No formal statistical analysis was performed.

\section{Noninvasive Diagnostics and Monitoring}

\section{Noninvasive Measurement of ICP with ONSD and Two- Depth Transcranial Doppler}

The development of noninvasive diagnostic methods to monitor ICP in patients with IIH could reduce the requirement for gold standard, invasive approaches such as lumbar puncture. A variety of noninvasive strategies for ICP monitoring have been studied including measurements of ONSD, TCD ultrasound, tympanic membrane displacement, magnetic resonance imaging (MRI), computed tomography (CT), pupillometry, visually evoked potentials, near-infrared spectroscopy, optoacoustic emission, venous ophthalmodynamometry, anterior fontanelle assessment, and electroencephalogram. ${ }^{19}$ Of these methods, ONSD and TCD, in particular two-depth TCD, appear to be the most extensively studied and most promising methods of noninvasive ICP monitoring. The measurement of the ONSD by use of ultrasonography, CT, or T2-weigted MRI has proven to be an effective and efficient way to measure ICP noninvasively as enlargement of the optic nerve head is a direct consequence of increased ICP since the optic nerve sheath is continuous with the meningeal layers. ONSD by ultrasonography provides valuable bedside assessment of ICP especially in the emergency setting. ${ }^{20}$ Recent studies using any of these three methods of ONSD measurement have demonstrated that the enlargement of ONSD in patients with elevated ICP or cerebral edema ranges from 4.58 to $6.9 \mathrm{~mm}$ versus 3.55 to 5.2 in healthy controls. These methods have been used to detect elevated ICP with sensitivity and specificity ranging from 84 to $98 \%$ and 42 to $92 \%$, respectively ( - Table $\mathbf{1}$ )..$^{21-36}$ The values can be compared with the results of two different meta-analyses from 2011 and 2015 that demonstrated the ability of ONSD ultrasonography to yield average sensitivity and specificity ranging from 90 to $95 \%$ and 85 to $92 \%$, respectively. ${ }^{29,30} \mathrm{~A}$ more recent meta-analysis found sensitivity and specificity to range from 88 to $95 \%$ and 74 to $96 \%$, respectively. ${ }^{31}$ 
Table 1 Studies evaluating the diagnostic value of ONSD techniques for measurement of ICP

\begin{tabular}{|c|c|c|c|c|c|}
\hline \multicolumn{6}{|c|}{ ONSD } \\
\hline Study, year & $n$ & Technique & Threshold (mm) & Sensitivity (\%) & Specificity (\%) \\
\hline Dubourg et al, 201129a & 231 & ONSD by ultrasound & $5-5.9$ & 90 & 85 \\
\hline Ohle et al, 201530a & 478 & ONSD by ultrasound & 5 & 96 & 92 \\
\hline Robba et al, 2018 $31 a$ & 320 & ONSD by ultrasound & $4.8-6.3$ & $88-94$ & $74-96$ \\
\hline Wang et al, $2015^{21}$ & 279 & ONSD by ultrasound & 4.2 & 95 & 92 \\
\hline Lee et al, $2016^{22}$ & 134 & ONSD by ultrasound & 5.5 & 99 & 85 \\
\hline Amini et al, $2013^{23}$ & 50 & ONSD by ultrasound & 5.5 & 100 & 100 \\
\hline Strumwasser et al, $2011^{35}$ & 10 & ONSD by ultrasound & - & 36 & 38 \\
\hline Salahuddin et, al $2016^{24}$ & 102 & ONSD by CT & 5.7 & 84 & 71 \\
\hline Sekhon et al, $2014^{25}$ & 57 & ONSD by CT & 6 & 97 & 42 \\
\hline Bekerman et al, $2016^{37}$ & 35 & ONSD by CT & 5.5 & - & - \\
\hline Vaiman et al, $2016^{28}$ & 443 & ONSD by CT & 5.5 & - & - \\
\hline Vaiman et al, $2016^{32}$ & 312 & ONSD by CT & 5.5 & - & - \\
\hline Geeraerts et al, $2008^{26}$ & 38 & ONSD by MRI & 5.82 & 90 & 92 \\
\hline Ozturk et al, $2017^{27}$ & 16 & ONSD by MRI & 5 & - & - \\
\hline Range & & & $4.2-6.3$ & $36-100$ & $38-100$ \\
\hline
\end{tabular}

Abbreviations: CT, computed tomography; ICP, intracranial pressure; MRI, magnetic resonance imaging; ONSD, optic nerve sheath diameter. andicates meta-analysis.

In testing for elevated ICP, Vaiman et al have proposed an ideal cutoff ONSD diameter of $5.5 \mathrm{~mm}^{32}$; yet, others have used cutoff diameters ranging from 4.1 to $6.0 \mathrm{~mm}$ ( - Table 1 ). In a retrospective study of patients specifically with $\mathrm{IIH}$, increased ONSD, defined by a cut-off diameter of $5.5 \mathrm{~mm}$, was identified in $94.3 \%$ of the study cohort and ONSD was found to be $6.21 .2 \mathrm{~mm}$ and $6.30 .9 \mathrm{~mm}$ in the right and left eye, respectively. ${ }^{33}$ Based upon these findings and the proposal made by Vaiman et al, use of ONSD with cutoff diameter of $5.5 \mathrm{~mm}$ is found to be adequate in detecting IIH with high sensitivity. Shofty et al also found ONSD to be enlarged in IIH patients versus healthy controls; however, these findings were described in a sample of children aged between 4 and $17 .{ }^{34}$ While ONSD has been proven effective in patients with IIH, Bekerman et al noted a potential limitation with the ONSD method in that, cases with narrow optic canal lumen $\left(<10 \mathrm{~mm}^{2}\right)$ result in false-negative tests and conclude that if the lumen of the canal is $<10 \mathrm{~mm}^{2}$, the ONSD method should not be used. ${ }^{37}$ It should also be noted that one study found the ONSD method of ICP measurement was unreliable, with sensitivity and specificity values of 36 and $38 \%$, respectively. ${ }^{35}$ Despite these findings as well as the fact that invasive ICP measurement via lumbar puncture remains the gold standard, measurement of ONSD has overall demonstrated efficacy and offers an efficient, cost-effective, noninvasive method of diagnosis and monitoring of ICP in patients with IIH.

TCD ultrasonography takes advantage of the relationship between ICP and the blood flow velocity in the middle cerebral artery. Multiple studies have exhibited a positive correlation between the TCD-derived pulsatility index (PI) and ICP with correlation coefficients ranging from 0.90 to 0.93 . $^{36,38}$ The sensitivity and specificity of TCD as a test of elevated ICP have been found to range from 81 to $88 \%$ and 96 to
97\%, respectively, using a PI cutoff range of 1.26 to $1.33 .^{36,39}$ Despite these findings, other studies indicate that PI is not a reliable predictor of elevated ICP. ${ }^{38,40}$ Zweifel et al do, however, specify that extreme values of PI may be useful in making the decision to undergo invasive ICP monitoring. ${ }^{41}$

While the use of standard TCD has not been proven to be a reliable method for ICP monitoring, two-depth TCD, which utilizes the simultaneous measurement of two different segments of the ophthalmic artery to determine an absolute value of ICP, may be more promising. This method involves simultaneous measurement of intracranial and extracranial blood flow velocity through the ophthalmic artery. While measurements are being made, an external pressure is applied to the extracranial segment of the artery. The external pressure at which the blood flow velocities equalize between the intracranial and extracranial segment is an accurate estimator of ICP. ${ }^{42}$ Sensitivity and specificity of the TCD and two-depth TCD approaches combined range from 68 to $89 \%$ and 84 to $97 \%$, respectively (- Table 2 ). ${ }^{36,38,39,43,44}$ One study comparing the two-depth approach versus invasive ICP monitoring demonstrated both accuracy with a low systematic error of $0.12 \mathrm{~mm}$ and precision with a standard deviation of recordings of $2.19 \mathrm{~mm} \mathrm{Hg} .{ }^{44}$ Additionally, Koskinen et al identified a positive correlation between invasive ICP monitoring and two-depth TCD ICP monitoring with $r=0.74 . .^{45}$ Interestingly, in a comparative analysis of two-depth TCD and ONSD, it was found that two-depth TCD displayed greater diagnostic reliability versus ONSD. The sensitivity and specificity were found to be 37 and $58 \%$, respectively, for ONSD and 68 and 84.3\%, respectively, for two-depth TCD. ${ }^{46}$ It should be noted that these findings for the ONSD group do not align with the previously discussed sensitivity and specificity ranges using this technique. Furthermore, there are 
Table 2 Studies evaluating the diagnostic value of TCD techniques for measurement of ICP

\begin{tabular}{|c|c|c|c|c|c|}
\hline \multicolumn{6}{|c|}{ TCD } \\
\hline Study, year & $n$ & Technique & Threshold (PI) & Sensitivity (\%) & Specificity (\%) \\
\hline Bellner et al, $2004^{36}$ & 81 & $\mathrm{TCD}$ & - & 89 & 92 \\
\hline Prunet et al, $2012^{43}$ & 20 & $\mathrm{TCD}$ & 1.35 & 80 & 90 \\
\hline Wakerly et al, $2015^{38}$ & 78 & TCD & 1.26 & 81 & 96 \\
\hline Wang et al, $2014^{39}$ & 93 & TCD & 1.34 & 89 & 97 \\
\hline Ragauskas et al, $2012^{44}$ & 85 & 2-depth TCD & - & 68 & 84 \\
\hline Range & & & $1.26-1.35$ & $68-89$ & 84-97 \\
\hline
\end{tabular}

Abbreviations: ICP, intracranial pressure; TCD, transcranial Doppler.

fewer studies conducted using the two-depth TCD method as compared with ONSD. Two-depth TCD also involved the use of an extracranial pressure apparatus limiting its ease of use especially as compared with normal TCD. ${ }^{47}$ However, two-depth TCD does retain the superior ability to record an absolute value of ICP and does not require a patient-specific calibration. Nonetheless, it is clear that more research and development are necessary before replacing the gold standard invasive ICP measurement strategies with noninvasive methods that can become a serious consideration.

\section{Radiographic Imaging Signs for IIH Diagnosis}

As previously discussed in the conventional diagnostic criteria for $\mathrm{IIH}$, radiographic imaging has, in the past, only been used for the purposes of diagnostic exclusion. Recent findings in the literature, however, suggest that specific radiographic signs, while not sufficient on their own, may provide significant support toward a diagnosis of IIH. A recent meta-analysis combining the results of 21 different studies with a total of 724 patients with IIH found transverse venous sinus stenosis to offer the greatest diagnostic reliability compared with other MRI signs and calculated a pooled sensitivity and specificity for transverse venous stenosis as an indicator of IIH of 84.4 and $94.9 \%$, respectively. MRI findings of "empty" sella, posterior displacement of pituitary stalk, meningoceles, posterior globe flattening, optic nerve head protrusion, optic nerve enhancement, optic nerve sheath distension, optic nerve tortuosity, slit-like ventricles, tight subarachnoid spaces, and inferior position of cerebellar tonsils demonstrated high specificity, but very low sensitivity as indicators of IIH. ${ }^{48}$ The idea that transverse venous sinus stenosis offers the best predictive value in the diagnosis of IIH as compared with other radiographic signs is strengthened by the identification of venous attenuation on CT in $96 \%$ of patients in one particular cohort of patients with $\mathrm{IIH}^{49}$

\section{Surgical Management Strategies}

\section{Optic Nerve Sheath Fenestration}

When the primary complaints are related to visual field and papilledema, ONSF is a valuable treatment option. A medial transconjunctival orbitotomy is performed and the retrobulbar portion of the optic nerve is exposed. Micropunctures are made in the optic nerve sheath to relieve CSF pressure within the optic nerve sheath compartment. ${ }^{50}$ ONSF allows for direct relief of CSF pressure in the optic nerve sheath making it a promising surgical intervention for patients with IIH who have failed medical treatment. Recent studies have demonstrated improvement or stability of visual acuity and visual fields in 86.2 to $94.4 \%$ and 86.7 to $95.9 \%$ of patients with $\mathrm{IIH}$, respectively. ${ }^{51-53}$ Yaqub et al also reported improved papilledema in $87.1 \%$ of IIH patients undergoing ONSF and indicated a significant change in papilledema as well as visual acuity from preoperative values. These findings can be compared with the results of four meta-analyses from 2007 to 2017 that calculated average improvement in visual acuity ranging from 59 to $85 \%$ of IIH patients undergoing ONSF ( - Table 3)..$^{171,184,55}$ Additionally, results of the meta-analyses showed average improvement in papilledema and headaches ranged from 80 to $81 \%$ and 44 to $81 \%$ of IIH patients undergoing ONSF, respectively ( - Tables 4 and 5 ). ${ }^{18,54-58}$ While there are few reports of major complications due to ONSF, common minor complications include diplopia, corneal dellen, and tonic pupil..$^{59}$ Several risk factors have been identified for ONSF failure and include CSF opening pressure of $>50 \mathrm{~cm}$ $\mathrm{H}_{2} \mathrm{O}$, male sex, poor visual acuity at presentation, and longer duration of symptoms before surgery. ${ }^{53,60}$ Despite potential minor complications and the risk of failure, ONSF provides an effective and relatively safe option for patients whose primary symptoms are related to visual acuity and papilledema.

\section{CSF Shunting}

Shunt procedures for the management of IIH provide a method of CSF shunting into the peritoneum to decrease ICP. The shunts are placed in the ventricles or the lumbar space and the CSF is directed to drain into the peritoneum. In a recent study, visual acuity, papilledema, and headaches were improved at a mean follow-up of 15 months in 93, 100, and $84 \%$, respectively, of IIH patients undergoing VP shunting. ${ }^{61}$ Meta-analyses conducted from 2007 to 2019 demonstrate that shunting, whether VP or LP, show improvement in visual acuity, papilledema, and headaches in an average of 38 to $56 \%, 70 \%$, and 62 to $80 \%$ of patients with $\mathrm{IIH}$, respectively (-Tables 3, 4 and 5). ${ }^{17,18,54-58}$ In consideration of the differential efficacy of VP versus LP shunts, one analysis reports that LP shunts are associated with a significantly increased likelihood of shunt revision and subsequent greater healthcare costs (\$3.7 vs. \$5.4 million) as compared with VP shunts. ${ }^{62}$ A more recent study, however, demonstrates statistically similar rates of shunt failure and complication between VP 
Table 3 Comparison of percentage of IIH patients with improved visual acuity symptoms by procedure type calculated by different meta-analyses

\begin{tabular}{|c|c|c|c|c|c|c|c|c|c|}
\hline \multirow[t]{2}{*}{ Study, year } & \multicolumn{3}{|c|}{ ONSF } & \multicolumn{3}{|c|}{ Shunt } & \multicolumn{3}{|c|}{ Stent } \\
\hline & $n$ & $\begin{array}{l}\text { MFU } \\
\text { (mo) }\end{array}$ & $\begin{array}{l}\text { VA } \\
\text { impr. }\end{array}$ & $n$ & MFU (mo) & VA impr. & $n$ & MFU (mo) & VA impr. \\
\hline Satti et al, $2015^{18}$ & 712 & 21 & 59 & 435 & 41 & 54 & 136 & 22 & 78 \\
\hline Lai et al, $2014^{54}$ & 332 & 38 & 67 & $\begin{array}{l}61 \text { (VP); } \\
287 \text { (LP) }\end{array}$ & 46 (VP); 48 (LP) & 49 (VP); 56 (LP) & 88 & 21 & 84 \\
\hline Feldon, $2007^{17}$ & 252 & 21 & 80 & $\begin{array}{l}31 \text { (VP); } \\
44 \text { (LP) }\end{array}$ & 48 (VP); 58 (LP) & 38 (VP); 44 (LP) & 17 & 11 & 47 \\
\hline Tarrats et al, $2017^{55}$ & 34 & - & 85 & - & - & - & - & - & - \\
\hline Range & & & $59-85$ & & & $38-56$ & & & $47-84$ \\
\hline
\end{tabular}

Abbreviations: IIH, idiopathic intracranial hypertension; impr., improvement; LP, lumboperitoneal; MFU, mean follow up; ONSF, optic nerve sheath fenestration; VA, visual acuity; VP, ventriculoperitoneal.

Table 4 Comparison of percentage of IIH patients with improved papilledema by procedure type calculated by different meta-analyses

\begin{tabular}{|l|l|l|l|l|l|l|l|l|l|}
\hline \multirow{2}{*}{ Study, year } & \multicolumn{4}{|c|}{ ONSF } & \multicolumn{4}{c|}{ Shunt } & \multicolumn{2}{c|}{ Stent } \\
\cline { 2 - 11 } & $n$ & $\begin{array}{l}\text { MFU } \\
\text { (mo) }\end{array}$ & $\begin{array}{l}\text { Papilledema } \\
\text { impr. }\end{array}$ & $n$ & $\begin{array}{l}\text { MFU } \\
\text { (mo) }\end{array}$ & $\begin{array}{l}\text { Papilledema } \\
\text { impr. }\end{array}$ & $n$ & $\begin{array}{l}\text { MFU } \\
\text { (mo) }\end{array}$ & $\begin{array}{l}\text { Papilledema } \\
\text { impr. }\end{array}$ \\
\hline Satti et al, 2015 & 712 & 21 & 80 & 435 & 41 & 70 & 136 & 22 & 83 \\
\hline $\begin{array}{l}\text { Tarrats et al, } \\
2017^{55}\end{array}$ & 34 & - & 81 & - & - & - & - & - & - \\
\hline $\begin{array}{l}\text { Nicholson et al, } \\
2019^{56}\end{array}$ & 474 & - & - & - & - & - & 474 & - & 93 \\
\hline $\begin{array}{l}\text { Starke et al, } \\
2015^{57}\end{array}$ & 185 & 22 & - & - & - & - & 185 & 22 & 94 \\
\hline $\begin{array}{l}\text { Puffer et al, } \\
2013^{58}\end{array}$ & 143 & 22 & - & - & - & - & 143 & 22 & 97 \\
\hline Range & & & $\mathbf{8 0 - 8 1}$ & & & $\mathbf{7 0}$ & & & $\mathbf{8 3 - 9 7}$ \\
\hline
\end{tabular}

Abbreviations: IIH, idiopathic intracranial hypertension; impr., improvement; MFU, mean follow up; ONSF, optic nerve sheath fenestration.

Table 5 Comparison of percentage of IIH patients with improved headache symptoms by procedure type calculated by different meta-analyses

\begin{tabular}{|c|c|c|c|c|c|c|c|c|c|}
\hline \multirow[t]{2}{*}{ Study, year } & \multicolumn{3}{|c|}{ ONSF } & \multicolumn{3}{|c|}{ Shunt } & \multicolumn{3}{|c|}{ Stent } \\
\hline & $n$ & MFU (mo) & $\begin{array}{l}\text { Headache } \\
\text { impr. }\end{array}$ & $n$ & $\begin{array}{l}\text { MFU } \\
\text { (mo) }\end{array}$ & $\begin{array}{l}\text { Headache } \\
\text { impr. }\end{array}$ & $n$ & $\begin{array}{l}\text { MFU } \\
\text { (mo) }\end{array}$ & $\begin{array}{l}\text { Headache } \\
\text { impr. }\end{array}$ \\
\hline Satti et al, $2015^{18}$ & 712 & 21 & 44 & 435 & 41 & 80 & 136 & 22 & 83 \\
\hline Lai et al, $2014^{54}$ & 332 & 38 & 36 & $\begin{array}{l}61 \text { (VP); } \\
287 \text { (LP) }\end{array}$ & $\begin{array}{l}46 \text { (VP); } \\
48 \text { (LP) }\end{array}$ & $\begin{array}{l}62(\mathrm{VP}) ; 75 \\
(\mathrm{LP})\end{array}$ & 88 & 21 & 82 \\
\hline Tarrats et al, $2017^{55}$ & 34 & - & 81 & - & - & - & - & - & - \\
\hline $\begin{array}{l}\text { Nicholson et al, } \\
2019^{56}\end{array}$ & 474 & - & - & - & - & - & 474 & - & 79 \\
\hline Starke et al, $2015^{57}$ & 185 & 22 & - & - & - & - & 185 & 22 & 78 \\
\hline Puffer et al, $2013^{58}$ & 143 & 22 & - & - & - & - & 143 & 22 & 88 \\
\hline Range & & & $44-81$ & & & $62-80$ & & & $78-88$ \\
\hline
\end{tabular}

Abbreviations: IIH, idiopathic intracranial hypertension; impr., improvement; LP, lumboperitoneal; MFU, mean follow up; ONSF, optic nerve sheath fenestration; VP, ventriculoperitoneal.

and LP shunts. ${ }^{63}$ One major problem with shunts for surgical management of IIH is the high rate of major complications that has been calculated to be $40.5 \%$ in a sample of 435 patients. ${ }^{18}$ Huang et al also demonstrated a decrease in functioning shunts over from 80 to $48 \%$ over 12 to 36 months post-surgery. ${ }^{64}$ Despite its apparent efficacy in reducing the symptoms of papilledema and headache in patients with $\mathrm{IIH}$, surgical shunting is also associated with a high rate of 
complication, decreased functioning overtime, and unclear efficacy in improving vision loss. The LP shunts with programmable valve systems are a potential alternative in avoiding brain injury, lower failure, and complication rates, lower incidence of intracranial hypotension, and flexibility in adjusting valve pressure settings postoperatively circumventing complications of under and over drainage. ${ }^{65,66}$

\section{Venous Sinus Stenting}

Venous sinus stenting is an emerging neurointerventional option for the surgical management that targets venous sinus stenosis in patients with $\mathrm{IIH} .{ }^{67}$ Using percutaneous transvenous access, sinusography is performed to measure the pressure gradient across the transverse sinus stenosis. If there is a pressure gradient of over $8 \mathrm{~cm}$ of water across the transverse sinus stenosis, the patient is placed on dual antiplatelets for 5 days. At a second sitting under general anesthesia, the stenosis is crossed using a microwire and microcatheter system over a roadmap obtained from carotid angiogram performed through a diagnostic catheter placed in the common carotid artery. Stenting with a self-expandable carotid stent of appropriate size and length is performed at the stenotic site ( - Fig. 1). Most of the lesions resolve after stenting and angioplasty is rarely used. After stenting, pressure measurements are repeated across the stented segment to document that the pressure gradient is nullified. The patient is followed up in the clinic with $\mathrm{CT}$ venography to document that the stent is patent (-Fig. 2) and is placed on monotherapy for antiplatelet medication. The most recent and comprehensive meta-analysis to date on the topic of transverse venous sinus stenting, which includes analysis of 474 patients from 20 studies, calculated, on average, that 93.7 and $79.6 \%$ of IIH patients undergoing the stenting procedure showed improvement in
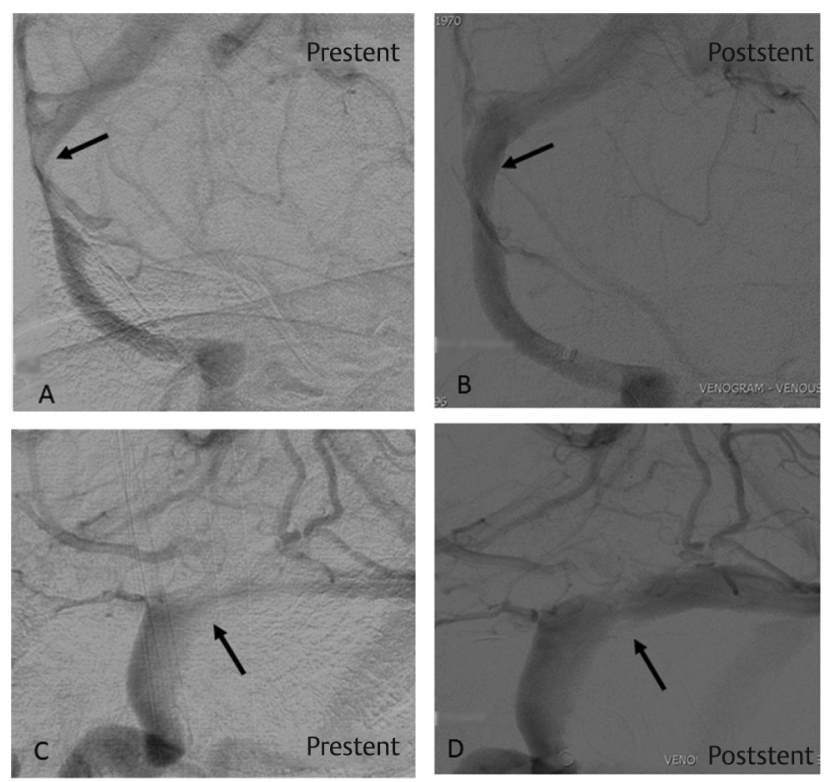

Fig. 1 (A-D) Digital subtraction angiography showing anteroposterior and lateral pre-stent images with narrowing (black arrows) of the right transverse-sigmoid sinus junction ( $\mathbf{A}$ and $\mathbf{C}$ ) and anteroposterior and lateral post-stent images showing improved caliber of the stenosis (black arrows) (B and $\mathbf{D}$ ).

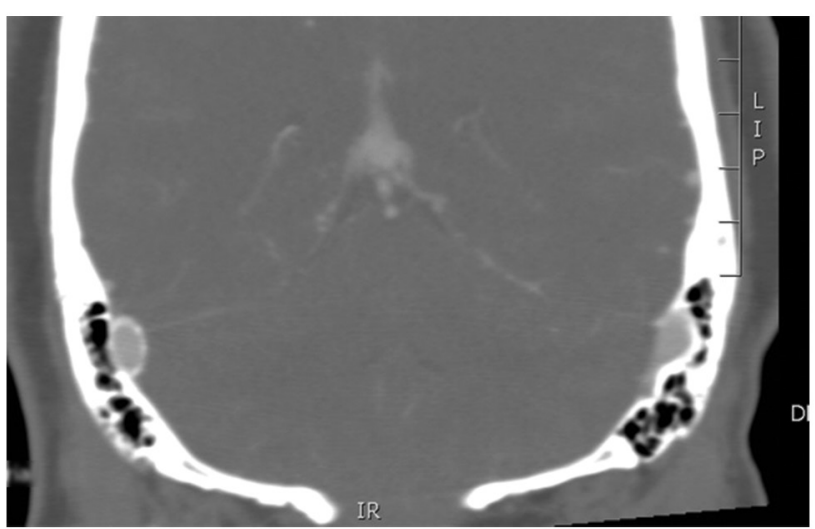

Fig. 2 Computed tomography venography of the same patient post-stenting on clinical follow-up after 6 weeks showing right transverse-sigmoid sinus stent to be widely patent (black arrow).

papilledema and headache, respectively. ${ }^{56}$ While the former study did not perform analysis of visual improvement, other meta-analyses have demonstrated an improvement in visual acuity, papilledema, and headache in an average of 47 to $84 \%$, 83 to $97 \%$, and 78 to $88 \%$ of IIH patients undergoing transverse venous sinus stenting, respectively (-Tables $\mathbf{3 - 5}$ ). ${ }^{17,18,54-58}$ It should be mentioned that one potential limitation of these findings is that these results are largely only applicable to those with patients who would have been documented with venous stenosis whereas those treated with ONSF or CSF shunting may or may not have demonstrated venous stenosis. Additional studies show venous stenting effectively reduces ICP immediately following the procedure and results in a significantly decreased CSF opening pressure for up to 3 months during the postoperative period. ${ }^{68,69}$ Potential complications occur at a rate of 1.6 to $7.4 \%$ and include occlusion of the vein of Labbe, subdural hematoma, and subarachnoid hemorrhage. ${ }^{18,56-58}$ This rate is lower than the identified rate of complication of $40.5 \%$ associated with CSF shunting identified by Satti et al and is suggested to contribute to the significantly lower financial cost of venous sinus stenting per 100 procedures as compared with CSF shunting. ${ }^{70}$ Risk factors for failed stent procedures include high CSF opening pressure preoperatively, female gender, and compression of the transverse sigmoid junction. ${ }^{71,72}$ There is some controversy whether venous sinus stenosis is a primary or secondary to IIH. Rohr et al presented three case reports where the patients developed a new stenosis of the transverse sinus proximal to the stent placement. ${ }^{73}$ Ducruey et al did, however, find that at follow-up of $30 \mathrm{IIH}$ patients undergoing transverse venous sinus stenting with mean follow-up of 23 months, all 30 patients exemplified patent stents demonstrating the long-term efficacy of the procedure. ${ }^{74}$ Occasionally, chronic cerebral venous sinus thrombosis may be seen associated with IIH. Few studies have evaluated the role of thrombolysis or mechanical thrombectomy along with venous sinus stenting in these cases and found it be an effective form of management. ${ }^{75,76}$ Transverse venous sinus stenting, therefore, offers a relatively safe and effective strategy for the improvement in visual acuity, headache, and papilledema in patients with IIH. In comparing 
different surgical options for $\mathrm{IIH}$, transverse venous sinus stenting appears to be an effective surgical management strategy in terms of ability to provide comprehensive symptom relief for patients with IIH.

\section{Conclusion}

The development and application of noninvasive diagnostic strategies and reliable neurointerventional management strategies for patients with IIH are important as healthcare shifts toward a focus on precision care and cost-effectiveness. While no noninvasive method can currently measure ICP with the same accuracy as invasive ICP monitoring, use of ONSD measurements and two-depth TCD ultrasonography offers potential as screening tools for ICP determination in settings lacking the invasive options. While not used for ICP monitoring, various radiographic imaging modalities may be useful in supporting the diagnosis of IIH. When considering surgical management of patients with $\mathrm{IIH}$, there is no clear optimal strategy. It appears that ONSF and shunting procedures are primarily associated with improvements in visual symptoms and headaches, respectively. Transverse venous sinus stenting demonstrates efficacy in improving visual acuity, papilledema, and headache with lower rates of complication than shunting. The choice of surgical management strategy should involve consideration of the individual patient's predominant signs and symptoms.

\section{Conflicts of Interest}

There are no conflicts of interest. Dr. Alon Harris would like to disclose that he receives remuneration from Shire, CIPLA, and AdOM for serving as a consultant. Dr. Harris also holds an ownership interest in AdOM and Oxymap. All relationships listed above are pursuant to Indiana University's policy on outside activities. None of the other authors listed have any financial disclosures.

\section{References}

1 McCluskey G, Doherty-Allan R, McCarron P, et al. Meta-analysis and systematic review of population-based epidemiological studies in idiopathic intracranial hypertension. Eur J Neurol 2018;25(10):1218-1227

2 Madriz Peralta G, Cestari DM. An update of idiopathic intracranial hypertension. Curr Opin Ophthalmol 2018;29(6):495-502

3 Chen JJ, Thurtell MJ, Longmuir RA, et al. Causes and prognosis of visual acuity loss at the time of initial presentation in idiopathic intracranial hypertension. Invest Ophthalmol Vis Sci 2015;56(6):3850-3859

4 Hatem CF, Yri HM, Sørensen AL, Wegener M, Jensen RH, Hamann S. Long-term visual outcome in a Danish population of patients with idiopathic intracranial hypertension. Acta Ophthalmol 2018;96(7):719-723

5 Yri HM, Wegener M, Sander B, Jensen R. Idiopathic intracranial hypertension is not benign: a long-term outcome study. J Neurol 2012;259(5):886-894

6 Rowe FJ, Sarkies NJ. Assessment of visual function in idiopathic intracranial hypertension: a prospective study. Eye (Lond) 1998;12(Pt 1) :111-118

7 Thambisetty M, Lavin PJ, Newman NJ, Biousse V. Fulminant idiopathic intracranial hypertension. Neurology 2007;68(3):229-232
8 McGeeney BE, Friedman DI. Pseudotumor cerebri pathophysiology. Headache 2014;54(3):445-458

9 Farb RI, Vanek I, Scott JN, et al. Idiopathic intracranial hypertension: the prevalence and morphology of sinovenous stenosis. Neurology 2003;60(9):1418-1424

10 Lee SC, Lueck CJ. Cerebrospinal fluid pressure in adults. J Neuroophthalmol 2014;34(3):278-283

11 Friedman DI, Liu GT, Digre KB. Revised diagnostic criteria for the pseudotumor cerebri syndrome in adults and children. Neurology 2013;81(13):1159-1165

12 Wall M, Kupersmith MJ, Kieburtz KD, et al; NORDIC Idiopathic Intracranial Hypertension Study Group. The idiopathic intracranial hypertension treatment trial: clinical profile at baseline. JAMA Neurol 2014;71(6):693-701

13 Handley JD, Baruah BP, Williams DM, Horner M, Barry J, Stephens JW. Bariatric surgery as a treatment for idiopathic intracranial hypertension: a systematic review. Surg Obes Relat Dis 2015;11(6):1396-1403

14 Koc F, Isik MR, Sefi-Yurdakul N. Weight reduction for a better visual outcome in idiopathic intracranial hypertension. Arq Bras Oftalmol 2018;81(1):18-23

15 Subramaniam S, Fletcher WA. Obesity and weight loss in idiopathic intracranial hypertension: a narrative review. J Neuroophthalmol 2017;37(2):197-205

16 NORDIC Idiopathic Intracranial Hypertension Study Group Writing Committee, Wall M, McDermott MP, et al. Effect of acetazolamide on visual function in patients with idiopathic intracranial hypertension and mild visual loss: the idiopathic intracranial hypertension treatment trial. JAMA 2014; 311(16):1641-1651

17 Feldon SE. Visual outcomes comparing surgical techniques for management of severe idiopathic intracranial hypertension. Neurosurg Focus 2007;23(5):E6

18 Satti SR, Leishangthem L, Chaudry MI. Meta-analysis of CSF diversion procedures and dural venous sinus stenting in the setting of medically refractory idiopathic intracranial hypertension. AJNR Am J Neuroradiol 2015;36(10):1899-1904

19 Khan MN, Shallwani H, Khan MU, Shamim MS. Noninvasive monitoring intracranial pressure - a review of available modalities. Surg Neurol Int 2017;8-51

20 Canakci Y, Koksal O, Durak VA. The value of bedside ocular ultrasound assessment of optic nerve sheath diameter in the detection of increased intracranial pressure in patients presenting to the emergency room with headache. Niger J Clin Pract 2018;21(6):778-782

21 Wang L, Feng L, Yao Y, et al. Optimal optic nerve sheath diameter threshold for the identification of elevated opening pressure on lumbar puncture in a Chinese population. PLoS One 2015;10(2):e0117939

22 Lee SU, Jeon JP, Lee H, et al. Optic nerve sheath diameter threshold by ocular ultrasonography for detection of increased intracranial pressure in Korean adult patients with brain lesions. Medicine (Baltimore) 2016;95(41):e5061

23 Amini A, Kariman H, Arhami Dolatabadi A, et al. Use of the sonographic diameter of optic nerve sheath to estimate intracranial pressure. Am J Emerg Med 2013;31(1):236-239

24 Salahuddin N, Mohamed A, Alharbi N, et al. The incidence of increased ICP in ICU patients with non-traumatic coma as diagnosed by ONSD and CT: a prospective cohort study. BMC Anesthesiol 2016;16(1):106

25 Sekhon MS, Griesdale DE, Robba C, et al. Optic nerve sheath diameter on computed tomography is correlated with simultaneously measured intracranial pressure in patients with severe traumatic brain injury. Intensive Care Med 2014;40(9):1267-1274

26 Geeraerts T, Newcombe VF, Coles JP, et al. Use of T2-weighted magnetic resonance imaging of the optic nerve sheath to detect raised intracranial pressure. Crit Care 2008;12(5):R114 
27 Ozturk Z, Atalay T, Arhan E, et al. The efficacy of orbital ultrasonography and magnetic resonance imaging findings with direct measurement of intracranial pressure in distinguishing papilledema from pseudopapilledema. Childs Nerv Syst 2017;33(9):1501-1507

28 Vaiman M, Sigal T, Kimiagar I, Bekerman I. Intracranial pressure assessment in traumatic head injury with hemorrhage via optic nerve sheath diameter. J Neurotrauma 2016;33(23):2147-2153

29 Dubourg J, Javouhey E, Geeraerts T, Messerer M, Kassai B. Ultrasonography of optic nerve sheath diameter for detection of raised intracranial pressure: a systematic review and meta-analysis. Intensive Care Med 2011;37(7):1059-1068

30 Ohle R, McIsaac SM, Woo MY, Perry JJ. Sonography of the optic nerve sheath diameter for detection of raised intracranial pressure compared to computed tomography: a systematic review and meta-analysis. J Ultrasound Med 2015;34(7):1285-1294

31 Robba C, Santori G, Czosnyka M, et al. Optic nerve sheath diameter measured sonographically as non-invasive estimator of intracranial pressure: a systematic review and meta-alysis. Intensive Care Med 2018;44(8):1284-1294

32 Vaiman M, Sigal T, Kimiagar I, Bekerman I. Noninvasive assessment of the intracranial pressure in non-traumatic intracranial hemorrhage. J Clin Neurosci 2016;34:177-181

33 Bekerman I, Sigal T, Kimiagar I, Almer ZE, Vaiman M. Diagnostic value of the optic nerve sheath diameter in pseudotumor cerebri. J Clin Neurosci 2016;30:106-109

34 Shofty B, Ben-Sira L, Constantini S, Freedman S, Kesler A. Optic nerve sheath diameter on MR imaging: establishment of norms and comparison of pediatric patients with idiopathic intracranial hypertension with healthy controls. AJNR Am J Neuroradiol 2012;33(2):366-369

35 Strumwasser A, Kwan RO, Yeung L, et al. Sonographic optic nerve sheath diameter as an estimate of intracranial pressure in adult trauma. J Surg Res 2011;170(2):265-271

36 Bellner J, Romner B, Reinstrup P, Kristiansson KA, Ryding E, Brandt L. Transcranial Doppler sonography pulsatility index (PI) reflects intracranial pressure (ICP) Surg Neurol 2004;62(1):45-51, discussion 51

37 Bekerman I, Kimiagar I, Sigal T, Vaiman M. Monitoring of intracranial pressure by CT-defined optic nerve sheath diameter. J Neuroimaging 2016;26(3):309-314

38 Wakerley BR, Kusuma Y, Yeo LL, et al. Usefulness of transcranial Doppler-derived cerebral hemodynamic parameters in the noninvasive assessment of intracranial pressure. J Neuroimaging 2015;25(1):111-116

39 Wang Y, Duan YY, Zhou HY, et al. Middle cerebral arterial flow changes on transcranial color and spectral Doppler sonography in patients with increased intracranial pressure. J Ultrasound Med 2014;33(12):2131-2136

40 Behrens A, Lenfeldt N, Ambarki K, Malm J, Eklund A, Koskinen LO. Transcranial Doppler pulsatility index: not an accurate method to assess intracranial pressure. Neurosurgery 2010;66(6):1050-1057

41 Hanlo PW, Gooskens RH, Nijhuis IJ, et al. Value of transcranial Doppler indices in predicting raised ICP in infantile hydrocephalus. A study with review of the literature. Childs Nerv Syst 1995;11(10):595-603

42 Zweifel C, Czosnyka M, Carrera E. de Riva N, Pickard JD, Smielewski P. Reliability of the blood flow velocity pulsatility index for assessment of intracranial and cerebral perfusion pressures in head-injured patients. Neurosurgery 2012;71(4):853-861

43 Prunet B, Asencio Y, Lacroix G, et al. Noninvasive detection of elevated intracranial pressure using a portable ultrasound system. Am J Emerg Med 2012;30(6):936-941
44 Ragauskas A, Matijosaitis V, Zakelis R, et al. Clinical assessment of noninvasive intracranial pressure absolute value measurement method. Neurology 2012;78(21):1684-1691

45 Koskinen LD, Malm J, Zakelis R, Bartusis L, Ragauskas A, Eklund A. Can intracranial pressure be measured non-invasively bedside using a two-depth Doppler-technique? J Clin Monit Comput 2017;31(2):459-467

46 Ragauskas A, Bartusis L, Piper I, et al. Improved diagnostic value of a TCD-based non-invasive ICP measurement method compared with the sonographic ONSD method for detecting elevated intracranial pressure. Neurol Res 2014;36(7):607-614

47 Siaudvytyte L, Januleviciene I, Ragauskas A, Bartusis L, Siesky $B$, Harris A. Update in intracranial pressure evaluation methods and translaminar pressure gradient role in glaucoma. Acta Ophthalmol 2015;93(1):9-15

48 Kwee RM, Kwee TC. Systematic review and meta-analysis of MRI signs for diagnosis of idiopathic intracranial hypertension. Eur J Radiol 2019;116:106-115

49 Ibrahim YA, Mironov O, Deif A, Mangla R, Almast J. Idiopathic intracranial hypertension: diagnostic accuracy of the transverse dural venous sinus attenuation on CT scans. Neuroradiol J 2014;27(6):665-670

50 Lagrèze WA, Gross N, Biermann J, Joachimsen L. [Indications and technique for transconjunctival optic nerve sheath fenestration: video article]. Ophthalmologe 2017;114(10):953-958

51 Moreau A, Lao KC, Farris BK. Optic nerve sheath decompression: a surgical technique with minimal operative complications. J Neuroophthalmol 2014;34(1):34-38

52 Obi EE, Lakhani BK, Burns J, Sampath R. Optic nerve sheath fenestration for idiopathic intracranial hypertension: a seven year review of visual outcomes in a tertiary centre. Clin Neurol Neurosurg 2015;137:94-101

53 Yaqub MA, Mehboob MA, Islam QU. Efficacy and safety of optic nerve sheath fenestration in patients with raised intracranial pressure. Pak J Med Sci 2017;33(2):471-475

54 Lai LT, Danesh-Meyer HV, Kaye AH. Visual outcomes and headache following interventions for idiopathic intracranial hypertension. J Clin Neurosci 2014;21(10):1670-1678

55 Tarrats L, Hernández G, Busquets JM, et al. Outcomes of endoscopic optic nerve decompression in patients with idiopathic intracranial hypertension. Int Forum Allergy Rhinol 2017;7(6):615-623

56 Nicholson P, Brinjikji W, Radovanovic I, et al. Venous sinus stenting for idiopathic intracranial hypertension: a systematic review and meta-analysis. J Neurointerv Surg 2019;11(4):380-385

57 Starke RM, Wang T, Ding D, et al. Endovascular treatment of venous sinus stenosis in idiopathic intracranial hypertension: complications, neurological outcomes, and radiographic results. ScientificWorldJournal 2015;2015:140408

58 Puffer RC, Mustafa W, Lanzino G. Venous sinus stenting for idiopathic intracranial hypertension: a review of the literature. J Neurointerv Surg 2013;5(5):483-486

59 Gilbert AL, Chwalisz B, Mallery R. Complications of optic nerve sheath fenestration as a treatment for idiopathic intracranial hypertension. Semin Ophthalmol 2018;33(1):36-41

60 Robinson ME, Moreau A, O'Meilia R, et al. The relationship between optic nerve sheath decompression failure and intracranial pressure in idiopathic intracranial hypertension. J Neuroophthalmol 2016;36(3):246-251

61 Bjornson A, Tapply I, Nabbanja E, et al. Ventriculo-peritoneal shunting is a safe and effective treatment for idiopathic intracranial hypertension. Br J Neurosurg 2019;33(1):62-70

62 Menger RP, Connor DE Jr, Thakur JD, et al. A comparison of lumboperitoneal and ventriculoperitoneal shunting for idiopathic intracranial hypertension: an analysis of economic impact and 
complications using the Nationwide Inpatient Sample. Neurosurg Focus 2014;37(5):E4

63 Azad TD, Zhang Y, Varshneya K, Veeravagu A, Ratliff JK, Li G. Lumboperitoneal and ventriculoperitoneal shunting for idiopathic intracranial hypertension demonstrate comparable failure and complication rates. Neurosurgery 2019; $\cdots$ : nyz080

64 Huang LC, Winter TW, Herro AM, et al. Ventriculoperitoneal shunt as a treatment of visual loss in idiopathic intracranial hypertension. J Neuroophthalmol 2014;34(3):223-228

65 Alkherayf F, Abou Al-Shaar H, Awad M. Management of idiopathic intracranial hypertension with a programmable lumboperitoneal shunt: early experience. Clin Neurol Neurosurg 2015;136:5-9

66 Nadkarni TD, Rekate HL, Wallace D. Concurrent use of a lumboperitoneal shunt with programmable valve and ventricular access device in the treatment of pseudotumor cerebri: review of 40 cases. J Neurosurg Pediatr 2008;2(1):19-24

67 Koovor JM, Lopez GV, Riley K, Tejada J. Transverse venous sinus stenting for idiopathic intracranial hypertension: Safety and feasibility. Neuroradiol J 2018;31(5):513-517

68 Matloob SA, Toma AK, Thompson SD, et al. Effect of venous stenting on intracranial pressure in idiopathic intracranial hypertension. Acta Neurochir (Wien) 2017;159(8):1429-1437

69 Patsalides A, Oliveira C, Wilcox J, et al. Venous sinus stenting lowers the intracranial pressure in patients with idiopathic intracranial hypertension. J Neurointerv Surg 2019;11(2):175-178

70 Ahmed RM, Zmudzki F, Parker GD, Owler BK, Halmagyi GM. Transverse sinus stenting for pseudotumor cerebri: a cost comparison with CSF shunting. AJNR Am J Neuroradiol 2014;35(5):952-958

71 Goodwin CR, Elder BD, Ward A, et al. Risk factors for failed transverse sinus stenting in pseudotumor cerebri patients. Clin Neurol Neurosurg 2014;127:75-78

72 Kumpe DA, Bennett JL, Seinfeld J, Pelak VS, Chawla A, Tierney M. Dural sinus stent placement for idiopathic intracranial hypertension. J Neurosurg 2012;116(3):538-548

73 Rohr A, Dörner L, Stingele R, Buhl R, Alfke K, Jansen O. Reversibility of venous sinus obstruction in idiopathic intracranial hypertension. AJNR Am J Neuroradiol 2007;28(4):656-659

74 Ducruet AF, Crowley RW, McDougall CG, Albuquerque FC. Long-term patency of venous sinus stents for idiopathic intracranial hypertension. J Neurointerv Surg 2014;6(3):238-242

75 Li K, Ren M, Meng R, et al. Efficacy of stenting in patients with cerebral venous sinus thrombosis-related cerebral venous sinus stenosis. J Neurointerv Surg 2019;11(3):307-312

76 He CZ, Ji XM, Wang LJ, et al. [Endovascular treatment for venous sinus stenosis in idiopathic intracranial hypertension]. Zhonghua Yi Xue Za Zhi 2012;92(11):748-751 